\title{
Lesão Renal Aguda Após Implantação Percutânea de Válvula Aórtica: Incidência, Fatores de Risco e Impacto na Mortalidade
}

\section{Acute Kidney Injury After Transcatheter Aortic Valve Implantation: Incidence, Risk Factors and Impact on Mortality}

\author{
Ana MARTINS ${ }^{1}$, Lurdes CASTRO ${ }^{1}$, Isabel FRAGATA ${ }^{1}$ \\ Acta Med Port 2020 Oct;33(10):664-674 - https://doi.org/10.20344/amp.11308
}

\section{RESUMO}

Introdução: A lesão renal aguda é uma complicação frequente após implantação percutânea de válvula aórtica com impacto na morbimortalidade dos doentes. É importante identificar fatores de risco modificáveis para desenvolver estratégias preventivas. Pretendeu-se determinar a incidência, fatores de risco e morbimortalidade a 30 dias e um ano relacionada com a ocorrência desta complicação em doentes submetidos a implantação percutânea de válvula aórtica.

Material e Métodos: Estudo retrospetivo em 149 doentes consecutivos submetidos a implantação percutânea de válvula aórtica no Hospital de Santa Marta. Os dados foram colhidos a partir dos registos periprocedimento, e estratificados pela existência de lesão renal aguda de acordo com a classificação AKIN.

Resultados: Um total de 149 doentes, 43,0\% sexo masculino com mediana de idade 82,00 [77,50 - 85,00] anos foram incluídos no estudo. A incidência de lesão renal aguda foi de $14,8 \%(n=22)$. O sexo feminino $(O R=, 138$, IC $95 \% ; 0,022-0,854 ; p=0,033)$ apresentou maior risco desta complicação. Os doentes com lesão renal aguda tiveram internamentos mais prolongados (OR 1,043 , IC $95 \% ; 1,001-1,085 ; p=0,043$ ); a mesma está associada ao aumento da mortalidade aos 30 dias (OR 13,889, IC 95\%; 2,371 - 81,363; $p=0,004)$

Discussão: O desenvolvimento de lesão renal aguda relaciona-se com variáveis pré-operatórias e é determinante da morbimortalidade. No entanto, o caráter retrospetivo e a reduzida dimensão da população estudada limitaram a capacidade de identificação e determinação do peso de cada um dos fatores

Conclusão: A lesão renal aguda é uma complicação da colocação percutânea de prótese aórtica com grande impacto na morbimortalidade, justificando a importância da sua prevenção.

Palavras-chave: Implante de Prótese de Válvula Cardíaca/efeitos adversos; Lesão Renal Aguda/etiologia; Substituição da Válvula Aórtica Transcateter; Válvula Aórtica/cirurgia

\section{ABSTRACT}

Introduction: Acute kidney injury is a frequent complication after transcatheter aortic valve implantation with great impact on morbidity and mortality. It is important to identify modifiable risk factors in order to develop preventive strategies. The aim of the study is to determine acute kidney injury incidence, risk factors and impact in patients that underwent transcatheter aortic valve implantation.

Material and Methods: Retrospective study in 149 consecutive patients that underwent transcatheter aortic valve implantation at Santa Marta Hospital. The data was collected from the periprocedural records and stratified by the occurrence of acute kidney injury according to the AKIN classification.

Results: A total of 149 patients, $43.0 \%$ male with median age 82.00 [77.50 - 85.00] years were included in the study. Incidence of acute kidney injury was $14.8 \%(n=22)$. Female patients (OR $0.138, \mathrm{Cl} 95 \% ; 0.022-0.854 ; p=0.033)$ had a higher risk of that complication. Patients with acute kidney injury had longer hospitalizations (OR 1.043, Cl 95\%; $1.001-1.085 ; p=0.043$ ); acute kidney injury was associated with increased 30 day mortality (OR 13.889, 95\% Cl; $2.371-81.363 ; p=0.004$ ).

Discussion: Acute kidney injury is associated with preprocedural variables and is a determinant of morbimortality. However, the retrospective character and the reduced sample size didn't allow the determination of the precise weight of each factor.

Conclusion: Acute kidney injury is a complication after transcatheter aortic valve implantation which played a significant role in morbimortality justifying its prevention.

Keywords: Acute Kidney Injury; Aortic Valve; Aortic Valve/surgery; Heart Valve Prosthesis Implantation/adverse effects; Risk Factors Acute Kidney Injury/etiology; Transcatheter Aortic Valve Replacement/adverse effects

\section{INTRODUÇÃO}

A lesão renal aguda (LRA) constitui uma complicação frequente após substituição endovascular da válvula aórtica (TAVI - transcatheter aortic valve implantation). ${ }^{1,2}$ Apresenta prevalência estimada de $3,4 \%-57,0 \%$, devendo-se esta heterogeneidade à utilização de diferentes definições de LRA. ${ }^{1,3-7}$ Encontra-se associada a elevada morbimorta- lidade a curto e longo prazo, com taxa de mortalidade aos 30 dias e um ano de $7,8 \%-29,0 \%$ e $10 \%-70 \%$, respetivamente. ${ }^{3,4,8}$

Os critérios Valve Academic Research Consortium-2 (VARC-2) adotaram a classificação AKIN para o diagnóstico de LRA estendendo-se o seu diagnóstico até ao séti-

1. Serviço de Anestesiologia. Hospital Santa Marta. Centro Hospitalar Universitário de Lisboa Central. Lisboa. Portugal.

$\triangle$ Autor correspondente: Ana Martins. ana.marga.vm@hotmail.com

Recebido: 19 de setembro de 2018 - Aceite: 10 de dezembro de 2019 | Copyright @ O Ordem dos Médicos 2020 
mo dia pós-procedimento de forma a garantir a associação com a realização da TAVI. ${ }^{9,10}$

Apesar da substituição valvular percutânea ser um procedimento minimamente invasivo que diminui a agressão e o stress cirúrgico, comparativamente à substituição da válvula aórtica por via aberta, as complicações pós-procedimento mantêm-se uma preocupação com interferência ao nível da morbimortalidade. , $^{2,3,6,11-14}$

Dados de um estudo nacional realizado na Alemanha demonstram uma discreta diminuição da prevalência de LRA nos doentes submetidos a TAVI ao longo dos anos $5,6 \%$ em 2007 e 5,2\% em $2013 .{ }^{4}$ O aumento da experiência na realização de TAVI's e o avanço tecnológico são igualmente aspetos a considerar e que podem ajudar a explicar a redução de LRA. ${ }^{4}$

Importa considerar que os doentes submetidos a TAVI apresentam características particulares no que respeita à idade, presença de comorbilidades e elevado risco cirúrgico que os tornam propensos para o desenvolvimento de LRA entre outras complicações com interferência ao nível da qualidade de vida. ${ }^{4-6,11}$ Além do mais, as características inerentes ao próprio procedimento influenciam o risco de desenvolvimento de LRA (o que se deve a aspetos técnicos e tipo de abordagem). ${ }^{3}$

A fisiopatologia da LRA é multifatorial. ${ }^{2,4,8,13,15,16}$ Vários fatores de risco periprocedimento têm vindo a ser identificados. Muitos dos fatores pré-procedimento são relacionados com o doente [idade, doença renal crónica (DRC), diabetes mellitus (DM), insuficiência cardíaca congestiva (ICC) e doença arterial periférica]. ${ }^{1,4,14,16,17}$ Alguns são específicos do procedimento - necessidade de administração de contraste, ocorrência de períodos de hipotensão extrema durante o pacing ventricular, manipulação de cateteres na aorta de doentes com doença aterosclerótica difusa (aumentando o risco de fenómenos de embolização) e via de inserção da válvula. ${ }^{1-4,6,8,9,13,15,18}$

O reconhecimento desses fatores constitui uma etapa fundamental na redução do risco de desenvolvimento desta complicação. ${ }^{3}$

Não existindo consenso sobre as medidas a adotar para prevenção da LRA na realização da TAVI, e com vista à sua redução é importante a implementação de um conjunto de estratégias preventivas dirigidas ao doente e que passam, em primeira instância, pela identificação dos doentes de elevado risco para a sua ocorrência e pela evicção dos fatores de risco. ${ }^{2,4,8} \mathrm{~A}$ otimização do volume intravascular do doente, redução do volume de contraste administrado, revisão das indicações de transfusão de produtos sanguíneos e a não administração de substâncias nefrotóxicas são medidas importantes na otimização do resultado dos doentes. ${ }^{2,3}$ Estas fazem parte do KDIGO bundle com benefício comprovado em cirurgia cardíaca. ${ }^{4,8,17}$

Ainda no âmbito da prevenção da LRA, têm sido realizados vários estudos que envolvem a administração de bicarbonato de sódio ou de $\mathrm{N}$-acetilcisteína sem evidência da sua eficácia. ${ }^{2,8}$

O sistema RenalGuard, o índice de resistência renal por doppler ou a utilização de dispositivos que permitam a proteção cerebrovascular de êmbolos durante a TAVI têm vindo a ser estudados e testados e poderão ser instrumentos úteis no futuro para a prevenção e identificação precoce de LRA..$^{4,8}$ Contudo, a sua utilização na prática clínica ainda não foi aceite. ${ }^{4}$

O ensaio POSEIDON (Prevention of Contrast Renal Injury With Different Hydratation Strategies) tem evidenciado a importância da medição da pressão tele-diastólica do ventrículo esquerdo para guiar a administração de fluidos nos doentes submetidos a TAVI enquanto medida de redução do risco relativo de LRA. ${ }^{8}$

As limitações inerentes às variáveis utilizadas no diagnóstico de LRA têm ainda levado à investigação de novos marcadores de disfunção renal perioperatória que possibilitem a identificação precoce deste síndrome e dos doentes de elevado risco. ${ }^{4,16,17}$ Contudo, os resultados referentes à eficácia dos mesmos são ainda limitados, razão pela qual a sua utilização ainda não foi implementada na prática clínica. ${ }^{4,16}$

Este trabalho teve como objetivos primários a determinação da incidência de LRA e identificação dos seus fatores de risco.

Como objetivo secundário pretendeu-se determinar o impacto do desenvolvimento desta complicação na morbimortalidade aos 30 dias e um ano nos doentes submetidos a TAVI.

\section{MATERIAL E MÉTODOS}

Realizou-se um estudo retrospetivo numa população de doentes submetidos a TAVI por via transfemoral ou subclávia no Hospital de Santa Marta entre os meses de janeiro de 2010 e dezembro de 2016, tendo-se considerado como doentes propostos para a realização deste procedimento todos aqueles que apresentavam estenose aórtica grave inoperável ou cujo risco cirúrgico era elevado.

Os dados foram colhidos a partir dos registos intra-procedimento e processo clínico do doente.

O trabalho não foi submetido a aprovação por Comissão de Ética por se tratar de um estudo observacional de caráter retrospetivo, com impossibilidade de obter o consentimento dos doentes. Acrescente-se que os doentes não foram submetidos a exames não habituais neste tipo de tratamento. Os dados colhidos pelos autores garantem a privacidade e confidencialidade dos doentes. Considera-se ainda o estudo com pertinência e interesse científico com vista à melhoria dos cuidados prestados nos doentes submetidos a TAVI.

$\mathrm{Na}$ análise de dados considerámos as características demográficas e ecocardiográficas dos doentes, abordagem anestésica, complicações periprocedimento e resultados a curto e médio prazo.

Os doentes com taxa de filtração glomerular $<60 \mathrm{~mL} /$ min foram considerados como tendo DRC.

Para a definição das complicações periprocedimento seguimos as orientações VARC-2, utilizando a classificação AKIN para diagnóstico de LRA. ${ }^{9}$ Todos os doentes 
classificados como estadio 1, 2 ou 3 da classificação AKIN foram considerados como tendo LRA.

Para a determinação de LRA foram utilizados os valores de creatinina pré-procedimento e os do sétimo dia pós-TAVI.

Foi recolhida informação referente à administração de $\mathrm{N}$-acetilcisteína e volume de contraste durante o intraprocedimento em todos os doentes. Foi avaliada correlação entre volume de contraste administrado e a duração do procedimento.

Não foi possível obter os dados referentes à fluidoterapia administrada durante o período periprocedimento.

Foi administrado a todos os doentes Ultravist ${ }^{\circledR}$, contraste de baixo peso molecular.

Para os doentes abordados em 2016, foi avaliada a mortalidade até à data da recolha dos dados (agosto 2018).

A análise e tratamento estatístico dos resultados foram feitos utilizando o programa informático SPSS $22,0^{\circledR}$.

Os doentes foram estratificados de acordo com a ocorrência ou não de LRA. São apresentadas as frequências absolutas e relativas de ocorrência para as variáveis e fatores de risco categóricos, e a média e desvio padrão ou mediana com intervalo interquartil (percentis 25 e 75, IIQ) nas variáveis quantitativas. As frequências da LRA em variáveis categóricas foram comparadas através do teste de qui-quadrado. O teste de Mann-Whitney foi utilizado para comparar as medianas das variáveis quantitativas. Este teste foi utilizado uma vez que a distribuição dos valores não apresentava uma distribuição normal (teste de Saphiro-Wilk, $p<0,05)$. As variáveis que, individualmente revelaram associação estatisticamente significativa com o desenvolvimento de LRA $(p<0,05)$, foram incluídas numa regressão logística para confirmar a sua importância e estimar o peso relativo de cada uma delas.

Os resultados foram considerados estatisticamente significativos se $p<0,05$.

\section{RESULTADOS}

A população de estudo incluiu 149 doentes, dos quais 64 são do sexo masculino (43,0\%), com idade mediana de $82,00[77,50-85,00]$ anos. Todos os doentes foram classificados como ASA III ( $n=54 ; 36,2 \%)$ ou IV ( $n=95 ; 63,8 \%)$.

A totalidade da amostra apresentava estenose aórtica grave como diagnóstico principal, razão pela qual foram submetidos a TAVI. A mesma foi realizada por via transfemoral na maioria dos doentes ( $n=145 ; 97,3 \%$ ), tendo os restantes sido abordados por via subclávia por má qualidade dos acessos femorais.

Verificou-se elevada prevalência de comorbilidades com $81,1 \%$ dos doentes a apresentar hipertensão arterial (HTA), 58,5\% dislipidemia, 100,0\% ICC e 40,4\% DRC. O valor mediano de creatinina pré-operatória foi de 0,970 $[0,800-1,265] \mathrm{mg} / \mathrm{dL}$.

Relativamente ao procedimento, o mesmo teve a duração mediana de 150,00 [120,00 - 180,00] minutos.

Os doentes foram monitorizados de acordo com as normas da American Society of Anesthesiologists (ASA).
Todos os doentes foram ainda monitorizados com índice biespetral. Os doentes submetidos a anestesia geral tiveram também monitorização com ecocardiografia transesofágica, sendo que nos submetidos a anestesia local com sedação a monitorização do procedimento foi feita com recurso a fluoroscopia e ecocardiografia transtorácica.

Foram colocados cateter venoso central, cateter arterial radial e eletrocateter para realização de pacing a todos os doentes.

Cada doente teve uma mediana de duração de internamento na unidade de cuidados intensivos ( $\mathrm{UCI}$ ) e total de $3,00[2,00-5,00]$ e $10,00[8,00-17,00]$ dias, respetivamente.

No que diz respeito à técnica anestésica, na maioria dos doentes foi realizada anestesia geral $(n=102 ; 68,5 \%)$. Nos restantes o procedimento decorreu sob anestesia local com sedação ( $n=47 ; 31,5 \%)$.

Verificaram-se complicações periprocedimento em $70,5 \%(n=105)$ dos casos. De entre as complicações mais frequentes, destacam-se as perdas sanguíneas intra-procedimento $(9,3 \%)$, a LRA $(3,1 \%)$, arritmias como a fibrilhação auricular $(8,6 \%)$, complicações vasculares (nomeadamente ao nível do membro inferior na abordagem transfemoral - $11,0 \%$ ) e eventos trombo-embólicos $(3,8 \%)$, todas elas determinantes para o resultado pós-operatório de cada doente.

A taxa de mortalidade aos 30 dias e um ano foi $4,0 \%$ e $7,4 \%$, respetivamente.

Nas Tabelas 1 a 3 apresentam-se as características demográficas e ecocardiográficas da população, bem como os dados periprocedimento.

A incidência de LRA foi de $14,8 \%(n=22)$ na amostra em estudo. A maioria foi classificada como estadio 1 (54,5\%), sendo igual a frequência dos estadios 2 e $3(22,7 \%)$.

Os doentes com LRA tendem a ser do sexo feminino ( $p$ $=0,011)$ e a apresentar doença arterial coronária (DAC) $(p$ $=0,020)$ - Tabela 1 .

Não se verificou diferença entre os grupos no que diz respeito a idade, classificação ASA, classificação EuroScore II ou outras patologias associadas (como HTA, DM, dislipidemia, ICC ou DRC prévia) - Tabela 1.

No que concerne aos dados periprocedimento, verificou-se que os doentes que desenvolveram LRA tendem a ter procedimentos $(p=0,017)$ e internamentos totais de maior duração $(p=0,025)$, maiores volumes de contraste administrado $(p=0,047)$ e maiores necessidades transfusionais $(p=0,001)$. O teste de correlação demonstrou que existe relação entre a maior duração do procedimento e o maior volume de contraste administrado ( $r$ de Pearson $0,245, p=0,04)$.

O número de doentes com alguma complicação periprocedimento foi superior no grupo de doentes com LRA ( $p$ $=0,023)$.

De entre as complicações mais frequentemente associadas com a ocorrência de LRA destaque para a hemorragia major $(p=0,003)$, complicações infeciosas $(p=0,002)$, insuficiência respiratória pós-procedimento $(p=0,010)$ ou 
Tabela 1 - Características gerais da população (pré-operatórias)

\begin{tabular}{|c|c|c|c|c|c|}
\hline \multicolumn{6}{|c|}{ Lesão renal } \\
\hline & \multirow{2}{*}{$\begin{array}{l}\text { Não LRA } \\
(n=127)\end{array}$} & \multicolumn{3}{|c|}{$\begin{array}{c}\text { LRA } \\
(n=22)\end{array}$} & \multirow[t]{2}{*}{ Comparação } \\
\hline & & Total & $\begin{array}{c}1 \\
(n=12)\end{array}$ & $\begin{array}{c}2 \text { ou } 3 \\
(n=10)\end{array}$ & \\
\hline Idade (anos), Mediana [IIQ] & $\begin{array}{c}82,0 \\
{[77,0-85,0]}\end{array}$ & $\begin{array}{c}81,5 \\
{[78,5-85,0]}\end{array}$ & $\begin{array}{c}83,5 \\
{[76,8-89,0]}\end{array}$ & $\begin{array}{c}80,50 \\
{[78,50-82,25]}\end{array}$ & $\begin{array}{c}\mathrm{MW}^{1}, \mathrm{U}=1354,5 \\
p=0,820\end{array}$ \\
\hline Sexo, n (\%) & & & & & $\begin{array}{c}\chi^{2[2]}=6,4636, \\
p=0,011^{*}\end{array}$ \\
\hline Masculino & $60(47,2)$ & $4(18,2)$ & $2(16,7)$ & $2(20,0)$ & \\
\hline Feminino & $67(52,8)$ & $18(81,8)$ & $10(83,3)$ & $8(80,0)$ & \\
\hline Classificação ASA, n (\%) & & & & & $\begin{array}{c}\chi^{2[2]}=0,219 \\
p=0,811\end{array}$ \\
\hline ASA 3 & $47(37,0)$ & $7(31,8)$ & $4(33,3)$ & $3(30,0)$ & \\
\hline ASA 4 & $80(63,0)$ & $15(68,2)$ & $8(66,7)$ & $7(70,0)$ & \\
\hline Classificação EuroScore II, n (\%) & & & & & $\begin{array}{c}\chi^{2[2]}=2,745 \\
p=0,253\end{array}$ \\
\hline Baixo: 0 - 2 & $12(9,4)$ & $3(13,6)$ & $1(8,3)$ & $2(20,0)$ & \\
\hline Moderado: 3 - 5 & $39(30,7)$ & $10(45,5)$ & $5(41,7)$ & $5(50,0)$ & \\
\hline Alto: $>5$ & $76(59,8)$ & $9(40,9)$ & $6(50,0)$ & $3(30,0)$ & \\
\hline HTA, n (\%) & & & & & $\begin{array}{c}\chi^{2[2]}=1,592 \\
p=0,253\end{array}$ \\
\hline Sim & $101(79,5)$ & $20(90,9)$ & $10(83,3)$ & $10(100,0)$ & \\
\hline Não & $26(20,5)$ & $2(9,1)$ & $2(16,7)$ & $0(0,0)$ & \\
\hline DM, n (\%) & & & & & $\begin{array}{c}\chi^{2[2]}=1,825 \\
p=0,205\end{array}$ \\
\hline Sim & $34(26,8)$ & $9(40,9)$ & $4(33,3)$ & $5(50,0)$ & \\
\hline Não & $93(73,2)$ & $13(59,1)$ & $8(66,7)$ & $5(50,0)$ & \\
\hline Dislipidemia, n (\%) & & & & & $\begin{array}{c}\chi^{2[2]}=0,157 \\
p=0,815\end{array}$ \\
\hline Sim & $75(59,1)$ & $12(54,5)$ & $5(41,7)$ & $7(70,0)$ & \\
\hline Não & $52(40,9)$ & $10(45,5)$ & $7(58,3)$ & $3(30,0)$ & \\
\hline Obesidade, n (\%) & & & & & $\begin{array}{c}\chi^{2[2]}=2,870 \\
p=0,135\end{array}$ \\
\hline Sim & $21(16,5)$ & $7(31,8)$ & $2(16,7)$ & $5(50,0)$ & \\
\hline Não & $106(83,5)$ & $15(68,2)$ & $10(83,3)$ & $5(50,0)$ & \\
\hline Tabagismo, n (\%) & & & & & $\begin{array}{c}\chi^{2[2]}=0,429 \\
p=1,000\end{array}$ \\
\hline Sim & $11(8,7)$ & $1(4,5)$ & $0(0,0)$ & $1(10,0)$ & \\
\hline Não & $116(91,3)$ & $21(95,5)$ & $12(100,0)$ & $9(90,0)$ & \\
\hline DPOC $^{3}, \mathrm{n}(\%)$ & & & & & $\begin{array}{c}\chi^{2[2]}=1,457 \\
p=0,237\end{array}$ \\
\hline Sim & $21(16,5)$ & $6(27,3)$ & $1(8,3)$ & $5(50,0)$ & \\
\hline Nâo & $106(83,5)$ & $16(72,7)$ & $11(91,7)$ & $5(50,0)$ & \\
\hline Fração ejeção, n (\%) & & & & & $\begin{array}{c}\chi^{2[2]}=1,451 \\
p=0,484\end{array}$ \\
\hline Boa $(\geq 50 \%)$ & $97(77,0)$ & $18(85,7)$ & $9(75,0)$ & $9(90,0)$ & \\
\hline Moderada $(40 \%-49 \%)$ & $18(14,3)$ & $1(4,8)$ & $2(16,7)$ & $0(0,0)$ & \\
\hline Má $(<40 \%)$ & $11(8,7)$ & $2(9,5)$ & $1(8,3)$ & $1(10,0)$ & \\
\hline
\end{tabular}

a ocorrência de paragem cardio-respiratória (PCR) $(p=$ $0,023)$ - Tabela 2.

A comparação entre a técnica anestésica ou a abordagem para realização de TAVI não evidenciou associação estatística com a ocorrência de LRA - Tabela 2.
$\mathrm{Na}$ análise multivariada, mantiveram associação estatísticamente significativa o sexo e a duração total de internamento (Tabela 3).

Face a estes resultados verifica-se que ser do sexo masculino é fator protetor para a ocorrência de LRA 
Tabela 1 - Características gerais da população (pré-operatórias)

\begin{tabular}{|c|c|c|c|c|c|}
\hline & & Lesãc & renal & & \\
\hline & Não LRA & & $\begin{array}{l}\text { LRA } \\
(n=22)\end{array}$ & & Comparação \\
\hline & $(n=127)$ & Total & $\begin{array}{c}1 \\
(n=12)\end{array}$ & $\begin{array}{l}2 \text { ou } 3 \\
(n=10)\end{array}$ & \\
\hline ICC NYHA ${ }^{4}, n(\%)$ & & & & & $\begin{array}{c}\chi^{2[2]}=2,345 \\
p=0,150\end{array}$ \\
\hline Classe I-II & $42(33,1)$ & $11(50,0)$ & $9(75,0)$ & $2(20,0)$ & \\
\hline Classe III-IV & $85(66,9)$ & $11(50,0)$ & $3(25,0)$ & $8(80,0)$ & \\
\hline $\begin{array}{l}\text { Creatinina pré-operatória }(\mathrm{mg} / \mathrm{dL}) \text {, } \\
\text { Mediana [IIQ] }\end{array}$ & $\begin{array}{c}0,970 \\
{[0,800-1,230]}\end{array}$ & $\begin{array}{c}0,970 \\
{[0,783-1,325]}\end{array}$ & $\begin{array}{c}1,100 \\
{[0,823-1,473]}\end{array}$ & $\begin{array}{c}0,900 \\
{[0,673-1,318]}\end{array}$ & $\begin{array}{c}\mathrm{MW}^{1}, \mathrm{U}=1333,0 \\
p=0,732\end{array}$ \\
\hline DRC prévia, n (\%) & & & & & $\begin{array}{c}\chi^{2[2]}=2,149 \\
p=0,162\end{array}$ \\
\hline Sim & $47(37,9)$ & $12(54,5)$ & $6(50,0)$ & $6(60,0)$ & \\
\hline Não & $77(62,1)$ & $10(45,5)$ & $6(50,0)$ & $4(40,0)$ & \\
\hline DVP $^{5}, \mathrm{n}(\%)$ & & & & & $\begin{array}{c}\chi^{2[2]}=0,016 \\
p=1,000\end{array}$ \\
\hline Sim & $33(26,0)$ & $6(27,3)$ & $3(25,0)$ & $3(30,0)$ & \\
\hline Não & $94(74,0)$ & $16(72,7)$ & $9(75,0)$ & $7(70,0)$ & \\
\hline DAC, n (\%) & & & & & $\begin{array}{l}\chi^{2[2]}=5,529 \\
\boldsymbol{p}=\mathbf{0 , 0 2 0 ^ { * }}\end{array}$ \\
\hline Sim & $57(44,9)$ & $4(18,2)$ & $3(25,0)$ & $1(10,0)$ & \\
\hline Não & $70(55,1)$ & $18(81,8)$ & $9(75,0)$ & $9(90,0)$ & \\
\hline EAM prévio, n (\%) & & & & & $\begin{array}{c}\chi^{2[2]}=0,064 \\
p=1,000\end{array}$ \\
\hline Sim & $20(15,7)$ & $3(13,6)$ & $2(16,7)$ & $1(10,0)$ & \\
\hline Não & $107(84,3)$ & $19(86,4)$ & $10(83,3)$ & $9(90,0)$ & \\
\hline $\mathrm{PCl}^{6}, \mathrm{n}(\%)$ & & & & & $\begin{array}{c}\chi^{2[2]}=1,592 \\
p=0,253\end{array}$ \\
\hline Sim & $26(20,5)$ & $2(9,1)$ & $2(16,7)$ & $0(0,0)$ & \\
\hline Não & $101(79,5)$ & $20(90,9)$ & $10(83,3)$ & $10(100,0)$ & \\
\hline Cirurgia cardíaca prévia, n (\%) & & & & & $\begin{array}{c}\chi^{2[2]}=0,987 \\
p=0,437\end{array}$ \\
\hline Sim & $36(28,3)$ & $4(18,2)$ & $1(8,3)$ & $3(30,0)$ & \\
\hline Não & $91(71,7)$ & $18(81,8)$ & $11(91,7)$ & $7(70,0)$ & \\
\hline CABG prévio ${ }^{7}, \mathrm{n}(\%)$ & & & & & $\begin{array}{c}\chi^{2[2]}=2,150 \\
p=0,168\end{array}$ \\
\hline $\operatorname{Sim}$ & $29(22,8)$ & $2(9,1)$ & $1(8,3)$ & $1(10,0)$ & \\
\hline Não & $98(77,2)$ & $20(90,9)$ & $11(91,7)$ & $9(90,0)$ & \\
\hline Arritmia, n (\%) & & & & & $\begin{array}{c}\chi^{2[2]}=0,566 \\
p=0,693\end{array}$ \\
\hline $\operatorname{Sim}$ & $12(9,4)$ & $1(4,5)$ & $1(8,3)$ & $0(0,0)$ & \\
\hline Nâo & $115(90,6)$ & $21(95,5 \%)$ & $11(91,7)$ & $10(100,0)$ & \\
\hline $\mathrm{FA}^{8}, \mathrm{n}(\%)$ & & & & & $\begin{array}{c}\chi^{2[2]}=0,708 \\
p=0,447\end{array}$ \\
\hline Sim & $35(27,6)$ & $8(36,4)$ & $1(8,3)$ & $7(70,0)$ & \\
\hline Não & $92(72,4)$ & $14(63,6)$ & $11(91,7)$ & $3(30,0)$ & \\
\hline $\begin{array}{l}\text { Hematócrito pré-operatório (\%), } \\
\text { Mediana [IIQ] }\end{array}$ & $\begin{array}{c}37,500 \\
{[34,400-40,100]}\end{array}$ & $\begin{array}{c}33,650 \\
{[30,775-41,850]}\end{array}$ & $\begin{array}{c}34,600 \\
{[32,075-42,700]}\end{array}$ & $\begin{array}{c}30,900 \\
{[27,750-41,550]}\end{array}$ & $\begin{array}{c}\mathrm{MW}^{1}: \mathrm{U}=1133,0 \\
p=0,158\end{array}$ \\
\hline
\end{tabular}

Os resultados foram considerados estatisticamente significativos se $p<0,05 ;{ }^{*}: p<0,05 ;{ }^{* *}: p<0.01$.

${ }^{[1]} \mathrm{MW}$ : teste de Mann-Withney; ${ }^{[2]} \chi^{2}$ : teste de qui-quadrado; ${ }^{3}$ DPOC: doença pulmonar obstrutiva crónica; ${ }^{4}$ NYHA: classificação New York Heart Association; ${ }^{5}$ DVP: doença vascular periférica;

${ }^{6} \mathrm{PCl}$ : percutaneous coronary intervention; ${ }^{7} \mathrm{CABG}$ : coronary artery bypass grafting; ${ }^{8} \mathrm{FA}$ : fibrilhação auricular

diminuindo em aproximadamente $86,0 \%$ o risco de desenvolver a complicação. É possível também verificar que a maior duração do internamento total aumenta o risco de desenvolver LRA, sendo o aumento de aproximadamente
4,0\% por cada dia de internamento.

$\mathrm{Na}$ análise univariada constatou-se ainda associação estatisticamente significativa entre LRA e mortalidade aos 30 dias $(p=0,005)$ e a um ano $(p=0,011)$ (Tabela 3$)$. 
Tabela 2 - Dados periprocedimento

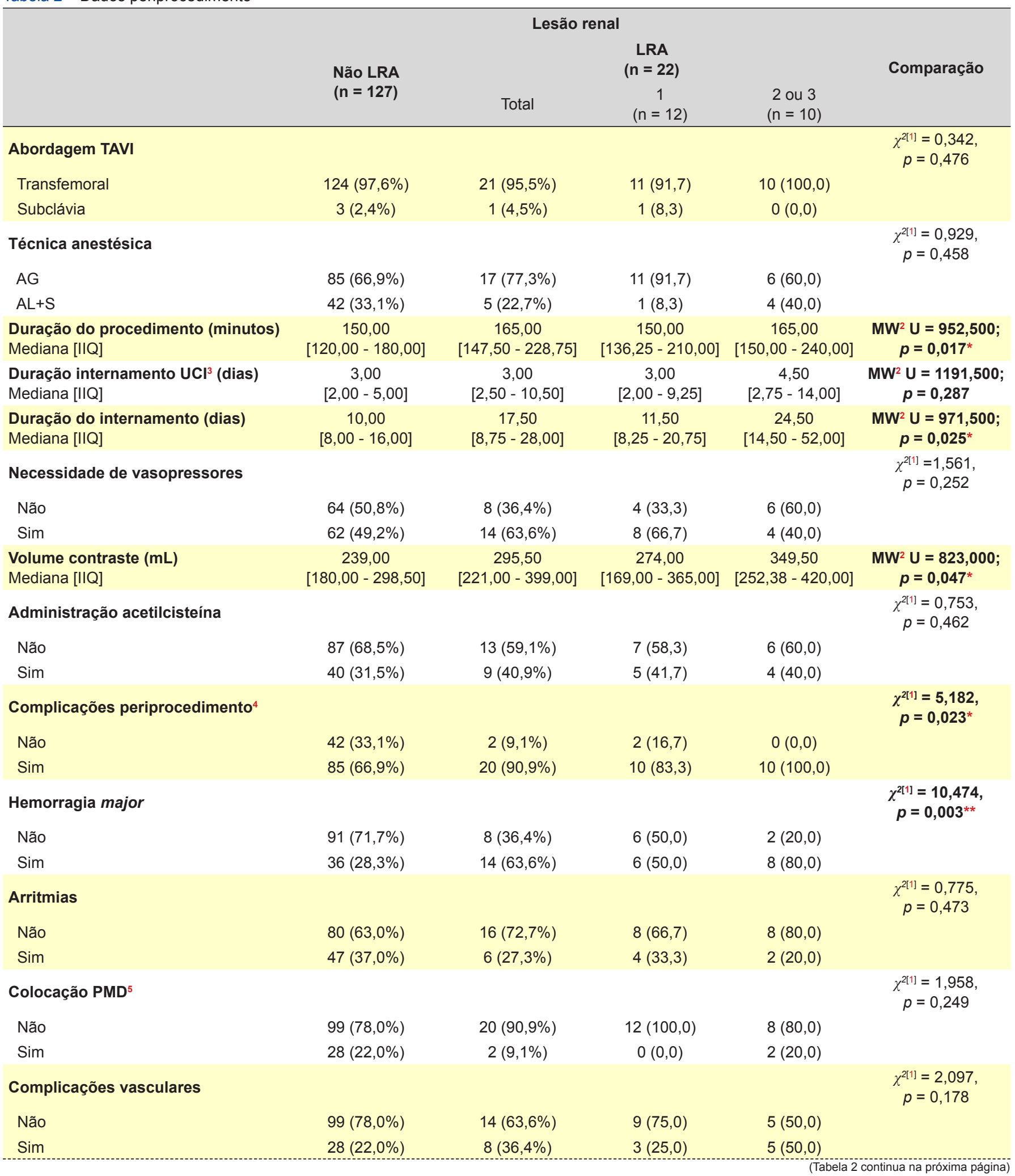

A LRA evidenciou ser um preditor independente de mortalidade a 30 dias $(p=0,005)$ em que os doentes com LRA apresentam risco acrescido de morte aos 30 dias de aproximadamente 13 vezes. A duração do procedimento foi identificado como outro dos preditores de mortalidade aos 30 dias $(p=0,002)$ aumentando $2,5 \%$ o risco de ocorrência do evento por cada minuto a mais de procedimento (Tabela 4).

Os resultados identificaram o tabagismo e a ocorrência de AVC pós-operatório como fatores protetores para a mortalidade aos 30 dias (Tabela 4).

Relativamente aos preditores de mortalidade a um ano, 
Tabela 2 - Dados periprocedimento

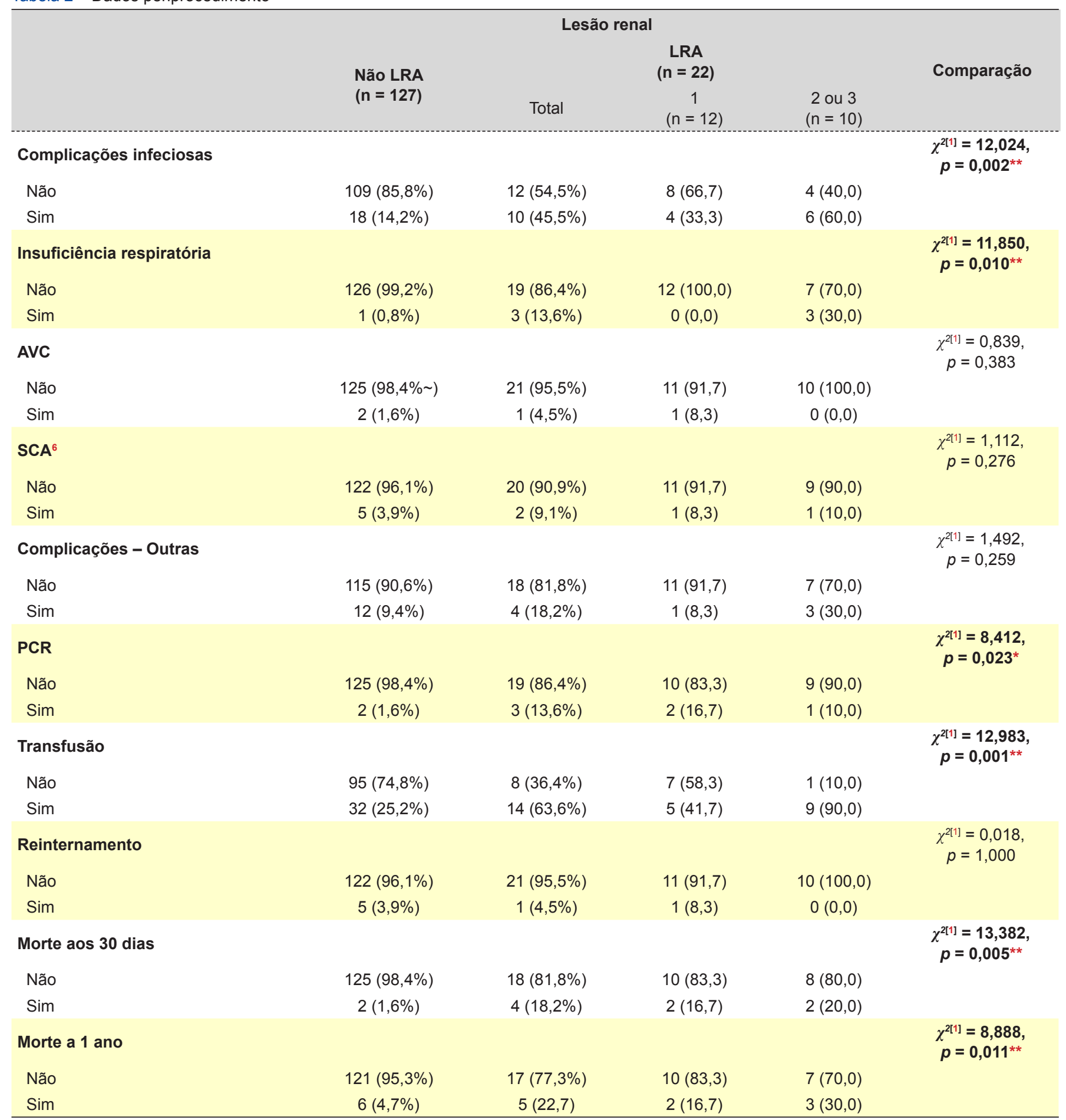

Os resultados foram considerados estatisticamente significativos se $p<0,05 ;{ }^{*}: p<0,05 ;{ }^{* *}: p<0.01$.

${ }^{[1]} \chi^{2}$ : Teste de qui-quadrado; ${ }^{[2]} \mathrm{MW}$ : teste de Mann-Withney; ${ }^{3} \mathrm{UCl}$ : unidade de Cuidados Intensivos; ${ }^{4}$ Definido como a ocorrência de qualquer complicação periprocedimento; ${ }^{5} \mathrm{PMD}$ : pacemaker definitivo; ${ }^{6}$ SCA: síndrome coronário agudo

os resultados apontam a presença de doença vascular periférica, a duração total de internamento, a ocorrência de hemorragia major e de LRA como variáveis que se incluem nesta categoria (Tabela 4). O aumento da duração do internamento contribui para o aumento do risco de mortalidade a um ano (5,0\% por cada dia de internamento); todas as restantes variáveis parecem ter um efeito protetor (Tabela 4).

\section{DISCUSSÃO}

A incidência de LRA na amostra em estudo e de acordo com os critérios VARC-2 foi de $14,8 \%$, valor que está dentro do intervalo previsto para a ocorrência desta complicação $(3,4 \%-57,0 \%)$. Outros estudos que utilizaram os critérios VARC-2 obtiveram valores semelhantes de LRA. ${ }^{11,12,19}$ Acredita-se que esta baixa incidência reflita algumas estratégias de proteção renal adotadas (longo intervalo de tempo entre 
Tabela 3 - Análise multivariada. Preditores independentes de LRA.

\begin{tabular}{|c|c|c|}
\hline Variáveis & $p$ & $\begin{array}{c}\text { OR } \\
\text { (IC 95\%) }\end{array}$ \\
\hline Sexo & $0,033^{* *}$ & $\begin{array}{c}0,138 \\
(0,022-0,854)\end{array}$ \\
\hline DAC & 0,054 & $\begin{array}{c}4,620 \\
(0,976-21,873)\end{array}$ \\
\hline Duração procedimento & 0,941 & $\begin{array}{c}1,000 \\
(0,987-1,014)\end{array}$ \\
\hline Dias de internamento & $0,043^{*}$ & $\begin{array}{c}1,043 \\
(1,001-1,085)\end{array}$ \\
\hline Volume contraste & 0,113 & $\begin{array}{c}1,005 \\
(0,999-1,012)\end{array}$ \\
\hline Complicações periprocedimento & 0,709 & $\begin{array}{c}1,441 \\
(0,212-9,795)\end{array}$ \\
\hline Hemorragia major & 0,065 & $\begin{array}{c}0,230 \\
(0,048-1,094)\end{array}$ \\
\hline Complicações infeciosas & 0,095 & $\begin{array}{c}0,270 \\
(0,058-1,256)\end{array}$ \\
\hline Insuficiência respiratória & 0,491 & $\begin{array}{c}0,144 \\
(0,001-35,918)\end{array}$ \\
\hline PCR & 0,246 & $\begin{array}{c}0,271 \\
(0,030-2,463)\end{array}$ \\
\hline Transfusão & 0,434 & $\begin{array}{c}0,555 \\
(0,127-2,424)\end{array}$ \\
\hline
\end{tabular}

Os resultados foram considerados estatisticamente significativos se $p<0,05 ;{ }^{*}: p<0,05 ;{ }^{* *}: p<0.01$.

a realização de angioplastia e a TAVI, fluidoterapia agressiva periprocedimento, administração de $\mathrm{N}$-acetilcisteína em alguns doentes, utilização de contraste isoosmolar em todos os doentes, evicção de fármacos nefrotóxicos e cuidado anestésico na manutenção da estabilidade hemodinâmica).

A aplicação dos critérios VARC-2 neste trabalho prendeu-se com a importância da uniformização da definição de LRA, o que torna possível uma melhor comparação e interpretação dos resultados obtidos, mas também com a elevada sensibilidade da classificação AKIN e com a possibilidade de identificar diferentes estadios de risco. ${ }^{11,14,19}$ Importa, no entanto, considerar que a classificação AKIN tem em conta os valores de creatinina e o débito urinário, ambos com várias limitações que podem acrescentar erros na identificação desta complicação. ${ }^{4,14}$

O sexo foi identificado como fator determinante para o desenvolvimento de LRA, tendo os indivíduos do sexo feminino apresentado maior risco de LRA na amostra. Apesar deste resultado poder dever-se ao maior número de elementos do sexo feminino, este é apontado como fator de risco em alguns estudos. ${ }^{4}$ No entanto, os resultados existentes são conflituosos, sendo dificíl estabelecer a relação entre sexo e risco de LRA. $^{8}$

Os resultados obtidos identificam também a duração total do internamento como fator de risco para a ocorrência de LRA. Contudo, face aos dados colhidos e à luz da prática clínica considera-se difícil estabelecer essa relação de causalidade. A associação estatisticamente significativa verificada entre o aumento da duração total de internamento e a ocorrência de LRA pode ser justificada com a ne- cessidade de resolução desta complicação, entre outras, já que o número de doentes com alguma complicação periprocedimento foi superior no grupo de doentes com LRA. Essa mesma associação está de acordo com resultados de estudos prévios que apontam o aumento da duração do internamento como consequência do desenvolvimento de LRA. ${ }^{1,6,7,16}$

A presença de DAC apresentou associação estatisticamente significativa com a ocorrência de LRA na análise univariada. Sabendo que a doença arterial oclusiva é um fator de risco para LRA, os resultados são concordantes com o esperado. 5,14 Vários estudos apontam o papel de outras comorbilidades no desenvolvimento de LRA (HTA, DM, ICC DRC) o que não se verificou no presente estudo. ${ }^{14,20}$ Dada a elevada prevalência destas comorbilidades na amostra analisada, tais resultados devem-se muito provavelmente à reduzida dimensão da amostra.

O volume de contraste administrado no grupo de doentes com LRA foi significativamente superior, o que se comprovou dever-se, pelo menos em parte, à maior duração da intervenção. O volume de contraste é um conhecido fator de risco para a ocorrência de LRA em que volumes superiores a $100 \mathrm{~mL}$ durante a realização de procedimentos coronários percutâneos têm sido associados ao desenvolvimento de nefropatia induzida por contraste. ${ }^{8,16} \mathrm{~A}$ mediana dos valores de volume de contraste administrado nos doentes que desenvolveram LRA foi cerca de três vezes superior ao estabelecido, o que torna evidente a necessidade da existência de esforços na tentativa de reduzir o volume de contraste administrado. A reduzida dimensão da amostra dificultou a determinação do limiar de contraste para a ocorrência de 
Tabela 4 - Preditores independentes de mortalidade a 30 dias e um ano

\begin{tabular}{|c|c|c|c|}
\hline Mortalidade & Variável & $p$ & $\begin{array}{c}\text { OR } \\
\text { (IC } 95 \%)\end{array}$ \\
\hline \multirow{4}{*}{30 Dias } & LRA & $0,004^{* *}$ & $\begin{array}{c}13,889 \\
(2,371-81,363)\end{array}$ \\
\hline & Duração procedimento & $0,002^{* *}$ & $\begin{array}{c}1,025 \\
(1,009-1,041)\end{array}$ \\
\hline & AVC & $0, \mathbf{0 4 3}^{*}$ & $\begin{array}{c}0,071 \\
(0,005-0,918)\end{array}$ \\
\hline & Tabagismo & $0,041^{*}$ & $\begin{array}{c}0,150 \\
(0,024-0,923)\end{array}$ \\
\hline \multirow{4}{*}{1 Ano } & Dias internamento & $0,007^{* *}$ & $\begin{array}{c}1,047 \\
(1,012-1,082)\end{array}$ \\
\hline & Hemorragia major & $0,038^{*}$ & $\begin{array}{c}0,259 \\
(0,072-0,930)\end{array}$ \\
\hline & DVP & $0,008^{* *}$ & $\begin{array}{c}0,173 \\
(0,047-0,627)\end{array}$ \\
\hline & LRA & $0,007^{* *}$ & $\begin{array}{c}0,169 \\
(0,046-0,613)\end{array}$ \\
\hline
\end{tabular}

Os resultados foram considerados estatisticamente significativos se $p<0,05 ;{ }^{*}: p<0,05 ;{ }^{* *}$ : $p<0.01$

LRA. Importa ainda reconhecer que a dificuldade em estabelecer este limiar e em isolar o efeito da administração de contraste relaciona-se com a presença de múltiplas comorbildades e outras variáveis inerentes ao próprio procedimento. É, no entanto, importante a existência de estudos que estabeleçam o valor de volume de contraste a partir do qual o risco de LRA aumente significativamente na população de doentes submetidos a TAVI. A ausência de associação estatisticamente significativa na análise multivariada entre o volume de contraste administrado e a LRA pode ainda ser explicada pela existência de outros fatores mais importantes para o desenvolvimento desta complicação. ${ }^{6,7,21}$

O papel da administração de $\mathrm{N}$-acetilcisteína na prevenção da LRA permanece ainda por esclarecer com resultados contraditórios nos diferentes estudos. ${ }^{2,18,22}$ Embora não seja uma medida protocolada, o fármaco foi administrado em alguns doentes em estudo. Os resultados não evidenciaram a influência da administração do fármaco no desenvolvimento de LRA ${ }^{23}$.

Verificou-se, de acordo com os resultados, uma tendência para a ocorrência de complicações totais periprocedimento e em particular de hemorragia major, ocorrência de transfusão sanguínea, complicações infeciosas, insuficiência respiratória e PCR no grupo de doentes com LRA. ${ }^{16}$

Reconhece-se o papel da ocorrência de hemorragia major no desenvolvimento de LRA, apontado como fator de risco em vários estudos, o que se deve muito provavelmente às consequências relacionadas com a diminuição do volume intravascular e consequente hipotensão, bem como à redução da capacidade de transporte de oxigénio. 1,12,24

A transfusão de produtos sanguíneos é apontado como outro fator de risco para a ocorrência de LRA., ${ }^{3,8,22}$ A associação estabelecida na análise univariada reforça a necessidade da existência de estratégias restritivas no que diz respeito à tranfusão de hemoderivados.

Vários estudos têm identificado a LRA como sendo forte preditor da mortalidade a curto e longo prazo. ${ }^{2,6,12,23}$ Os resultados enfatizam a ocorrência de LRA enquanto fator de risco para a mortalidade aos 30 dias após TAVI, sendo esta associação dependente de características da população e das complicações periprocedimento. ${ }^{22}$ Pode encarar-se a LRA como um marcador de falência multisistémica, de doentes mais frágeis ou de procedimentos mais complexos que contribui para a mortalidade dos doentes. ${ }^{12,23} \mathrm{~A}$ complexidade dos procedimentos juntamente com o maior volume de contraste administrado são certamente variáveis que contribuem para a maior duração do procedimento e que justificam que este seja fator determinante da mortalidade aos 30 dias.

A duração total do internamento foi a única variável encontrada como sendo determinante do aumento da mortalidade a um ano, o que pode dever-se a possível associação com a ocorrência de complicações periprocedimento ou até mesmo contexto de falência multiorgânica em que se tenha desenvolvido $\mathrm{LRA}^{18}$. Difícil de explicar são sem dúvida os resultados que evidenciam o caráter protetor do tabagismo e da ocorrência de AVC pós-operatório para a mortalidade aos 30 dias, ou da ocorrência de hemorragia major, DVP ou LRA como fatores protetores da mortalidade a um ano. Os fatores atrás referidos, por comprometerem a perfusão renal, têm sido evidenciados como fatores de risco em estudos prévios. ${ }^{18}$ Atribui-se por isso estes resultados à reduzida dimensão da amostra como fator limitante para uma análise multivariada com forte poder preditor. ${ }^{21}$

O presente estudo apresenta algumas limitações que dizem respeito ao seu caráter retrospetivo e reduzida dimensão da amostra e também ao facto de analisar dados de um único centro.

A impossibilidade da análise detalhada das tendências hemodinâmicas constitui outra limitação. A hipotensão intra-procedimento frequentemente associada ao pacing ventricular durante a implantação valvular encontra-se associada ao desenvolvimento de LRA o que se deve à 
elevada sensibilidade às alterações tensionais por parte do $\operatorname{rim}^{3,5,23}$

A não utilização do critério da medição do débito urinário para determinação de LRA, contemplada nos critérios VARC-2 deve-se ao facto de não ter sido possível aferir o mesmo de forma precisa na totalidade dos doentes. ${ }^{10}$

Importa acrescentar a possível existência de efeito confundidor de variáveis como a gravidade da estenose aórtica, área valvular, o gradiente médio de pressão entre outras variáveis ecográficas assim como o tipo de válvula aórtica utilizada em cada doente. ${ }^{14,21}$

É de realçar ainda que os dados dizem respeito ao início da implementação da TAVI no nosso hospital, sendo de esperar que a incidência desta complicação diminua com o aumento da experiência na realização do procedimento e com o alargamento dos critérios de realização de TAVI's em população de doentes com estenose aórtica com menor risco cirúrgico. ${ }^{13,21}$

Acreditamos que este trabalho ajude a complementar os estudos realizados até ao momento e reflita o que tem vindo a ser feito no Hospital de Santa Marta no âmbito de um programa TAVI e com especial enfoque numa complicação - a LRA. Tem como objetivo último a elaboração de protocolo com base na evidência científica disponível de forma a orientar a prática clínica na abordagem multidisciplinar dos doentes propostos para TAVI no que concerne à prevenção da LRA.

\section{CONCLUSÃO}

A incidência de LRA na amostra em estudo foi de 14,8\%.

De acordo com os resultados obtidos, o sexo feminino parece ser fator de risco para o desenvolvimento de LRA. A ocorrência desta complicação contribui de forma significativa para o aumento da duração de internamento bem como da mortalidade aos 30 dias e um ano.

\section{REFERÊNCIAS}

1. Filho A, Katz M, Campos C, Carvalho L, Siqueira D, Tumelero R, et al. Impact of acute kidney injury on short- and long-term outcomes after transcatheter aortic valve implantation. Rev Esp Cardiol. 2019;72:21-9.

2. Saia F, Ciuca C, Taglieri N, Marrozzini C, Savini C, Bordoni B, et al. Acute kidney injury following transcatheter aortic valve implantation: incidence, predictors and clinical outcome. Int J Cardiol. 2013;168:103440.

3. Scherner M, Wahlers T. Acute kidney injury after transcatheter aortic valve implantation. J Thorac Dis. 2015;7:1527-35.

4. Zaleska-Kociecka M, Dabrowski M, Stepinska J. Acute kidney injury after transcatheter aortic valve replacement in the elderly: outcomes and risk management. Clin Interv Aging. 2019;14:195-201.

5. Chatani K, Abdel-Wahab M, Wubken-Kleinfeld N, Gordian K, Potzing K, Mostafa A, et al. Acute kidney injury after transcatheter aortic valve implantation: Impact of contrast agents, predictive factors, and prognostic importance in 203 patients with long-term follow-up. J Cardiol. 2015;66:514-9.

6. Gebauer K, Diller GP, Kaleschke G, Kerckhoff G, Malyar N, Meyborg M, et al. The risk of acute kidney injury and its impact on 30-day and longterm mortality after transcatheter aortic valve implantation. Int J Nephrol. 2012;2012:483748.

7. Catalano M, Lin D, Cassiere H, Kohn N, Rutkin B, Maurer G, et al. Incidence of acute kidney injury in patients with chronic renal insufficiency: transcatheter versus surgical aortic valve replacement. J Interv Cardiol. 2019;2019:9780415.
A associação encontrada entre LRA e o volume de contraste administrado, várias complicações periprocedimento e aumento da duração do procedimento realçam a influência da primeira na morbilidade dos doentes.

A LRA demonstrou ser um preditor da mortalidade a 30 dias.

Face ao impacto evidenciado da LRA na morbimortalidade dos doentes, eleva-se a importância do desenvolvimento de scores de risco que permitam identificar e estratificar no período pré-operatório os doentes com maior risco de desenvolver LRA. A adoção de estratégias preventivas integradas numa abordagem multidisciplinar e individualizada que passem pela monitorização hemodinâmica periprocedimento e que permitam a deteção precoce de situações de redução de volume intravascular ou de baixo débito cardíaco são fundamentais.

\section{PROTECÇÃO DE PESSOAS E ANIMAIS}

Os autores declaram que os procedimentos seguidos estavam de acordo com os regulamentos estabelecidos pelos responsáveis da Comissão de Investigação Clínica e Ética e de acordo com a Declaração de Helsínquia da Associação Médica Mundial emitida em 2013.

\section{CONFIDENCIALIDADE DOS DADOS}

Os autores declaram ter seguido os protocolos do seu centro de trabalho acerca da publicação de dados.

\section{CONFLITOS DE INTERESSE}

Os autores declaram não ter conflitos de interesses relacionados com o presente trabalho.

\section{FONTES DE FINANCIAMENTO}

Os autores declaram que este trabalho não recebeu qualquer tipo de suporte financeiro de nenhuma entidade no domínio público ou privado.

8. Ram P, Mezue K, Pressman G, Rangaswami J. Acute kidney injury posttranscatheter aortic valve replacement. Clin Cardiol. 2017;40:1357-62.

9. Elhmidi Y, Bleiziffer S, Deutsch M, Krane M, Mazzitelli D, Lange R, et al. Acute kidney injury after transcatjeter aortic valve implantation: incidence, predictors and impact on mortality. Arch Cardiovasc Dis. 2014;107:133-9

10. Kappetein AP, Head SJ, Généreux P, Piazza N, Mieghuem NM, Blackstone $\mathrm{EH}$, et al. Updated standardized endpoint definitions for transcatheter aortic valve implantation: the Valve Academic Research Consortium-2 consensus document. J Thorac Cardiovasc Surg. 2013;145:6-23.

11. Konigstein $M$, Ben-Assa $E$, Abramowitz $Y$, Steinvil A, Rubinow EL, Havanuk O, et al. Usefulness of Updated Valve Academic Research Consortium-2 Criteria for acute kidney injury following transcatheter aortic valve implantation. Am J Cardiol. 2013;112:1807-11.

12. Konigstein M, Ben-Assa E, Banai S, Shacham $Y$, Ziv-Baran T, Abramowitz Y, et al. Periprocedural bleeding, acute kidney injury, and long-term mortality after transcatheter aortic valve implantation. Can J Cardiol. 2015;31:52-6.

13. Villablanca P, Mathew V, Thourani V, Rodés-Cabau J, Bangalore S, Makkiya $M$, et al. A meta-analysis and meta-regression of long-term outcomes of transcatheter versus surgical aortic valve replacement for severe aortic stenosis. Int J Cardiol. 2016;225:234-43.

14. Wang J, Yu W, Zhou Y, Yang Y, Li C, Liu N, et al. Independent risk factors contributing to acute kidney injury according to Updated Valve 
Academic Research Consortium-2 Criteria after transcatheter aortic valve implantation: a metaanalysis and meta-regression of 13 studies. J Cardiothorac Vasc Anesthe. 2017;31:816-26.

15. Zivkovic N, Elbaz-Greener G, Quu F, Arbel Y, Cheema AN, Dvir D, et al. Bedside risk score for prediction of acute kidney injury after transcatheter aortic valve replacement. Open Heart. 2018;5:e000777.

16. Najjar M, Salna M, George I. Acute kidney injury after aortic valve replacement: incidence, risk factors and outcomes. Expert Rev Cardiovasc Ther. 2015;13:301-6.

17. Romagnoli S, Ricci Z, Ronco C. Perioperative acute kidney injury: prevention, early recognition, and supportive measures. Nephron. 2018;140:105-10.

18. Gargiuloa G, Sanninoa A, Capodannob D, Perrinoa C, Capranzanob $\mathrm{P}$, Barbantib $\mathrm{M}$, et al. Impact of postoperative acute kidney injury on clinical outcomes after TAVI: a meta-analysis of 5,971 patients. Catheter Cardiovasc Interv. 2015;86:518-27.

19. Koifman E, Segev A, Fefer P, Barbash I, Sabbag A, Medvedovsky $D$, et al. Comparison of acute kidney injury classifications in patients undergoing transcatheter aortic valve implantation: predictors and long- term outcomes. Catheter Cardiovasc Interv. 2015;87:523-31.

20. Bagur R, Webb J, Nietlispach F, Dumont E, Larochellière R, Doyle D, et al. Acute kidney injury following transcatheter aortic valve implantation: predictive factors, prognostic value, and comparison with surgical aortic valve replacement.European Heart J. 2010;31:865-74.

21. Généreux P, Kodali S, Green P, Paradiz J, Daneault B, Rene G, et al. Incidence and effect of acute kidney injury after transcatheter aortic valve replacement using the new Valve Academic Research Consortium Criteria. Am J Cardiol. 2013;111:100-5.

22. Mei M, Zhao H, Pan Q, Pu Y, Tang M, Shen B. Efficacy of N-Acetylcysteine in preventing acute kidney injury after cardiac surgery: a metaanalysis study. J Invest Surg. 2017;31:14-23.

23. Goebel N, Baumbach H, Ahad S, Voehringer M, Hill S, Albert M, et al. Transcatheter aortic valve replacement: does kidney function affect outcome? Ann Thorac Surgy. 2013;96:507-12.

24. Wang J, Yu W, Jin Q, Li Y, Liu N, Hou X, et al. Risk factors for postTAVI bleeding according to the VARC-2 bleeding definition and impact of the bleeding on short-term mortality: a meta-analysis. Can J Cardiol. 2017;33:525-34. 